\title{
Diagnostics of the Core Mechanical Construction Based on Vibroacoustic Measurements and Distribution of Temperature Field in Transformer Magnetic Circuit
}

\author{
S. BORUCKI*, A. Cichon AND T. BOCZAR \\ Faculty of Electrical Engineering, Automatic Control and Computer Science, Opole University of Technology, \\ Prószkowska 76, 45-758 Opole, Poland
}

(Received May 9, 2015; in final form July 20, 2015)

\begin{abstract}
Subject matter of this article concerns the results of research works on the development and use of the vibroacoustic method to evaluate technical state of transformer cores. The article presents obtained measurement results of transformer mechanical vibration for two cases of its operation: with twisted and loosened core. In order to demonstrate negative effects of operating a transformer with a loosened core, this publication presents thermographic images showing temperature distribution on its magnetic core. The article characterises the examined transformer, employed measurement system and methodology of the completed experiment. The vibroacoustic and thermographic measurements were carried out during idle work of the transformer. Core vibration was registered for three measurement axes: $0 X, 0 Y$ and $0 Z$. Completed tests have proven that loosened screws pressing the core cause evident increase in the value of magnetic core vibration acceleration. At the same time, the temperature rises by more than $25 \%$.
\end{abstract}

DOI: $10.12693 /$ APhysPolA.128.306

PACS: 43.40.-r, 43.40.Vn, 43.58.Wc

\section{Introduction}

The quality and continuity of electric energy supplies to its consumers depend on reliable power system operation. Among its essential components there are power transformers, which allow combining networks characterised by different voltage levels. Stable operation of the whole transmission and distribution system largely depends on failure-free work of this equipment. Compared to other electrical equipment, power transformers are characteristic of little fallibility [1-3], however their breakdowns, especially of disastrous character, carry serious consequences of technical and economic nature [4-6].

One of the reasons for breakdowns of power transformers is the loss of rigidity of their core structure. Elements affecting the development of defects of this type are magnetostrictive vibrations and cellulose depolymerisation. Their negative effect is the loss of mechanical and electric strength of paper and pressboard insulation. Magnetostriction and depolymerisation cause gradual loosening of magnetic circuit sheet packs, which brings about considerable increase in the number of no-load losses, local overheating and the rise of electroinsulating oil temperature in defect occurrence location. Many months of the transformer operation with progressing core structure defect not only significantly accelerates insulating system ageing processes, but may also cause abrupt increase in the concentration of flammable gases, and emergency equipment shut-down by gas-flow protection.

* corresponding author; e-mail: s.borucki@po.opole.pl
The subject matter of this article concerns developing the non-invasive method for transformer core diagnostics, which is based on the measurements and analysis of vibroacoustic signals measured at idle work of the monitored power engineering facility. The examined problems constitute an extension of scientific and research works carried out at Opole University of Technology on the application and effective use of vibroacoustic methods in industrial conditions. The results of research carried out in this area were discussed e.g. in publications [7-15]. The main cognitive purpose of completed studies, results of which are demonstrated in this article, is to show negative effects of operation of transformers with loosened mechanical structure of the core. Completed research allowed proving that loosened screws pressing transformer core cause distinct increase in the effective value of acceleration of mechanical vibrations in its magnetic circuit, and its temperature rise by more than $25 \%$.

\section{Methodology of carried out experiment}

The vibroacoustic and thermographic parameters of the transformer core were measured and analysed at the Laboratory for Insulating Systems Diagnostics at Opole University of Technology. Experimental examinations covered the low-voltage transformer with dry insulation, supplied with $400 \mathrm{~V}$ rated voltage. Vibrations and temperature field distributions for magnetic circuit of the monitored unit were recorded while working at idle, without any transformer loading. Table I contains the main technical parameters of the examined transformer.

The choice of a dry transformer for laboratory tests allowed relatively "easy" modelling of its magnetic circuit 
TABLE I

Main technical parameters of the examined transformer.

\begin{tabular}{c|c}
\hline \hline Technical parameter & Examined transformer \\
\hline manufacturer & Elhand Lubliniec \\
type & ET3S-15 \\
power [kVA] & 15 \\
voltages $[\mathrm{V} / \mathrm{V}]$ & $400 / 24$ \\
vector group & Yy \\
manufacture year & 2006
\end{tabular}

defect. The modelling involved reducing the moment of torsional force pressing the core. In order to eliminate the interferences during core vibration measurements (resulting from vibrations in the transformer windings), defects of coils were not simulated - they were twisted from the factory torque compressive forces.

The transformer vibroacoustic signals were carried out using a triaxial accelerometer (4524-B-001 type) manufactured by Bruel\&Kjael, with sensitivity of $10 \mathrm{mV} / \mathrm{g}$. The measuring transducer was fitted at " $\mathrm{P}$ " on upper yoke beam of the core (Fig. 1).

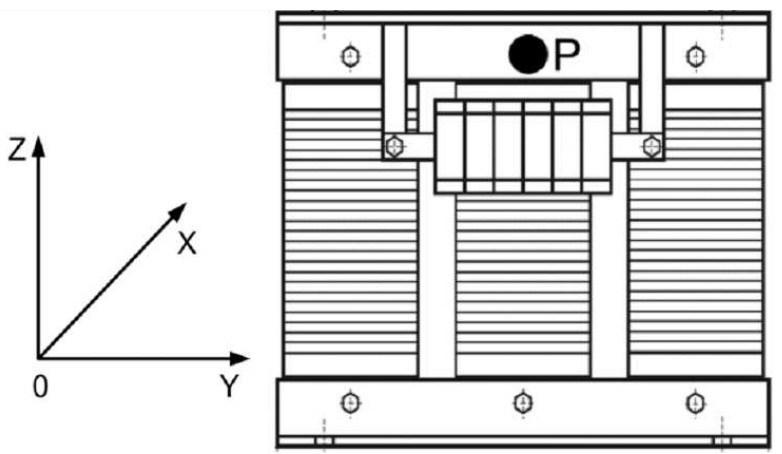

Fig. 1. Drawing of the examined transformer with marked accelerometer fixing point and measurement axes for core mechanical vibrations.

Vibroacoustic signals received by the sensor and generated by the core magnetostrictive vibrations in three measurement axes $(0 X, 0 Y, 0 Z)$ were given as an input for the Pulse LAN-XI system manufactured by Bruel\&Kjael. The Pulse Time Data Recorder and Pulse LabShop software was used to record and analyse the measured vibrations. Before starting the recording procedure, measuring tracks each time were calibrated using vibration calibrator of the frequency $f=159.15 \mathrm{~Hz}$ $\pm 0.02 \%$ and acceleration $g=10 \mathrm{~m} / \mathrm{s}^{2} \pm 3 \%$.

The measurements of the core temperature fields distribution for the states of work of magnetic circuit in the diagnosed transformer analysed as part of this publication, were carried out using a portable thermographic camera, type of ThermoGear G100EX, with sensitivity of $0.04^{\circ} \mathrm{C}$, manufactured by NEC.

The assessment of the impact of modelled transformer core defect on the assigned vibroacoustic parameters and relevant thermographic images was carried out in three experiment stages. The first stage concerned recording of temperature distribution for magnetic circuit of the examined unit without supply voltage; determination of the so-called initial core temperature (reference temperature). The next stage of tests involved transformer activation (idle work) with correctly twisted magnetic core, and then thermographic and vibroacoustic measurements were carried out after core temperature stabilisation (after $5 \mathrm{~h}$ of work). The last stage of carried out experiment involved magnetic core defect modelling and bringing back the supply voltage. In this case, thermographic measurements and recording of mechanical vibrations were carried out following the next core temperature stabilisation, that is approximately after another $5 \mathrm{~h}$ of the examined transformer operation. Obtained results of completed analysis of measured vibroacoustic signals and distributions of the core temperature fields are shown in the following section.

\section{Analysis of obtained results}

The vibrations of magnetic circuit in the examined transformer, measured in three measurement axes were analysed using the effective value of vibration acceleration and trajectories of amplitude spectrum within 5$3200 \mathrm{~Hz}$. The changes in its core surface temperature caused by the modelled defect were evaluated on the basis of the analysis of determined thermographic images.

Table II contains a synthetic comparison of effective values of the core mechanical vibrations acceleration, which was determined in three measurement axes, in two analysed cases of the transformer operation: with correctly twisted core and loosened core.

TABLE II

Comparison of effective values of the core vibrations acceleration, determined for three measurement axes and for two cases of the examined transformer operation.

\begin{tabular}{c|c|c}
\hline \hline \multirow{2}{*}{ Measurement axis } & \multicolumn{2}{|c}{$\begin{array}{c}\text { The RMS value of the acceleration } \\
\text { in the band } 5-3200 \mathrm{~Hz}\left[\mathrm{~mm} / \mathrm{s}^{2}\right]\end{array}$} \\
\cline { 2 - 3 } & Twisted core & Loosened core \\
\hline $0 X$ & 28.6 & 98.4 \\
$0 Y$ & 13.8 & 36.6 \\
$0 Z$ & 12.6 & 48.2
\end{tabular}

Figure 2 illustrates the changes in frequency shares for individual vibration harmonics recorded in the $0 X$ axis. The characteristic marked with letter (a) concerns the results obtained during the unit operation with correctly twisted core, whereas letter (b) - the unit with loosened magnetic core structure. Other amplitude spectra trajectories (shown in Figs. 3 and 4) apply to the analysis of vibrations recorded in axes $0 Y$ and $0 Z$, respectively.

Completed analysis of changes in the effective value of transformer vibration acceleration showed that modelling of magnetic circuit damage brought about evident 

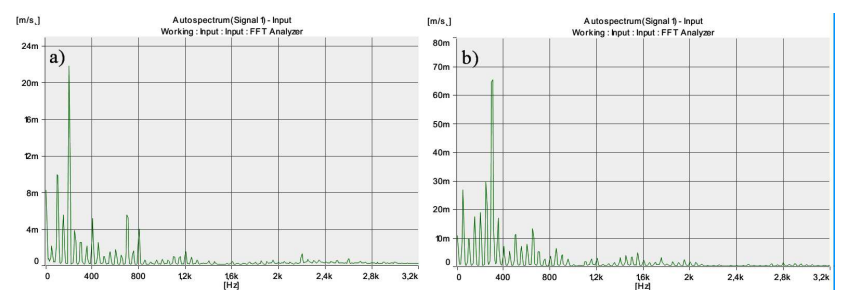

Fig. 2. Example amplitude spectra of transformer core vibrations, recorded in measurement axis $0 X$ : (a) twisted core, (b) loosened core.
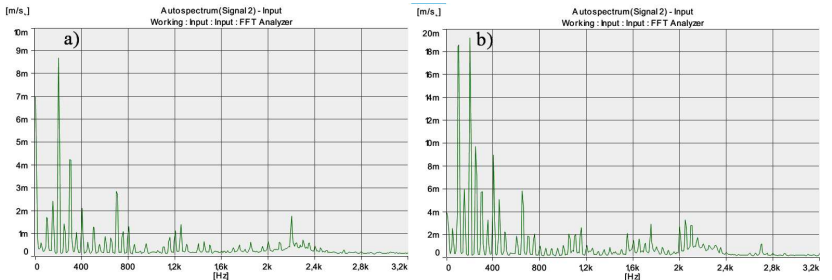

Fig. 3. As in Fig. 2, but for $O Y$ axis.

increase of vibration level for the whole unit. This phenomenon is observed at the same time in all measurement axes, independently of the direction of force pressing the core. Therefore, it means that using a uniaxial accelerometer and fixing it only in one measurement axis also allows carrying out an effective magnetic circuit diagnostics, and thus makes it possible to purchase much cheaper transducer and measuring equipment.

Spectral analysis of the measured vibroacoustic signals (Figs. 2-4) have proven that loosening of screws twisting upper and lower yoke (loss of rigidity of the core mechanical structure) in particular causes increase in the share of vibration harmonics within $5-1000 \mathrm{~Hz}$. Thus, completed research confirms that the transformer magnetic circuit loosening does not largely translate into the increase of frequency components from the band over $1000 \mathrm{~Hz}$. This effect is visible for the recording and analysis of results obtained in all three measurement axes: $0 X, 0 Y, 0 Z$.

The analysis of changes in transformer temperature following its core damage is a complement and possibly one of the most important results of the completed research. The illustration below presents thermographic images of magnetic circuit in the diagnosed unit obtained during the discussed experiment. Figure 5a concerns determination of an initial state that is core temperature in a switched off transformer. Thermogram no. 5b presents temperature distribution for a unit working without core
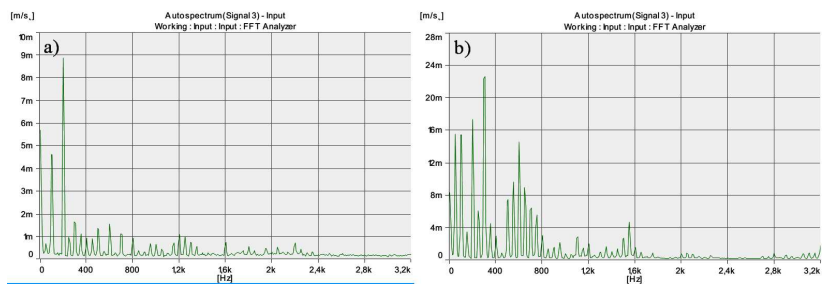

Fig. 4. As in Fig. 2, but for $O Z$ axis. defect, and Fig. 5c concerns operation of the transformer with loosened magnetic circuit. Thermographic images shown in Fig.5 concern stabilised operating conditions of the examined transformer - magnetic core temperature fluctuations did not exceed $0.2^{\circ} \mathrm{C}$.

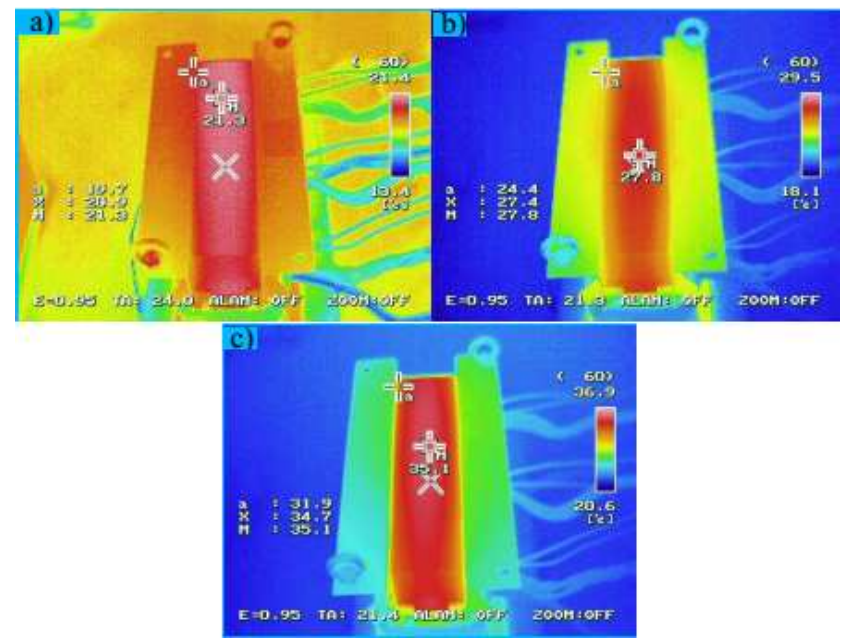

Fig. 5. Thermograms of transformer magnetic circuit: (a) unit switched off, (b) transformer operation with twisted core, (c) transformer operation with loosened core.

The completed thermographic examinations give grounds to state that the core of transformer working idle for $5 \mathrm{~h}$ without modelled magnetic circuit defect becomes heated to the temperature of $c a .27 .8^{\circ} \mathrm{C}$ (Fig. $5 \mathrm{~b}$ ). Whereas, operation of the same unit for another $5 \mathrm{~h}$, but with mechanical structure defect in its core, brings about magnetic core temperature rise up to ca. $35.1{ }^{\circ} \mathrm{C}$, and thus the temperature rise exceeds $25 \%$ (Fig. $5 \mathrm{c}$ ).

In case of the low-power transformer (15 kVA only) analysed for the purposes of this article, obtained core temperature rise is still not dangerous for insulating system of this power engineering facility. Nevertheless, it should be emphasised that the examined transformer was working with no load and without any extra core heating by current flowing through windings. In case of loading with rated current, obtained distributions of temperature fields and temperature in the so-called hot spots would be much higher for this unit.

\section{Summary}

Completed analysis of effective value of acceleration and amplitude spectrum of the transformer core vibrations provided grounds to prove evident differences in the specified vibroacoustic indicators. Effective acceleration values and the values of individual harmonic components for vibrations within $5 \div 3200 \mathrm{~Hz}$, determined for the simulated core defect, first of all differ in the amplitude and ranges of the so-called prevailing frequencies. These differences are visible for the recording made in each of the three measurement axes (Table II, 
Figs. 2-4). The completed spectral analysis (Figs. 2-4) has also proven that the transformer magnetic circuit loosening primarily causes the increase in harmonic amplitude within $5-1000 \mathrm{~Hz}$.

Complete research has proven that the work of power transformer with loosened mechanical structure of the core brings about an evident temperature rise in its active part (Fig. 5). For low-power unit used during laboratory tests this temperature rise exceeded $25 \%$. Therefore, carried out model experiment allows showing how serious problem it might be to operate high-power transformers $(S>100 \mathrm{MVA})$ with developing and identified too late defect in their cores.

The tests carried out by the authors of this work (their results are presented also in this publication) have proven that the vibroacoustic method may be used for noninvasive diagnostics of power transformer cores. Observation of changes in time of acceleration effective value and shares of harmonics in the spectrum of measured vibrations allows efficient evaluation of the technical state of magnetic circuit mechanical structure in transformers, especially those of strategic importance.

\section{Acknowledgments}

The work financed with the means from the National Research and Development Center within LIDER program.

\section{References}

[1] V. Mijailović, El. Power Syst. Res. 80, 987 (2010).

[2] H. Ding, R. Heywood, J. Lapworth, S. Ryder, in: Int. Conf. Euro TechCon 2009, Stretton 2009, p. 1.
[3] S.S. Rajurkar, A.R. Kulkami, J.G. Nandapurkar, in: Proc. 16th National Power Syst. Conf., India, 2010, p. 180.

[4] A.J.M. Cardoso, L.M.R. Oliveira, Int. J. Comadem 2, 5 (1999).

[5] CIGRE-WG 12-05, An International Survey on Failures in Large Power Transformers in Service, Electra 88, 21 (1983).

[6] T. Suwanasri, J. Haema, R. Rhadungthin, C. Suwanarsi, in: ECTI-CON 2009, Int. Conf. 1, 18 (2009).

[7] S. Borucki, IEEE Trans. Power Deliv. 27, 670 (2012).

[8] S. Borucki, Acta Phys. Pol. A 120, 571 (2011).

[9] S. Borucki, A. Cichoń, Przeglad Elektrotechniczny 86, 45 (2010) (in Polish).

[10] P. Frącz, Acta Phys. Pol. A 120, 604 (2011).

[11] M. Szmechta, T. Boczar, P. Frącz, Acta Phys. Pol. A 120, 744 (2011).

[12] A. Cichoń, P. Frącz, D. Zmarzły, Acta Phys. Pol. A 120, 585 (2011).

[13] D. Wotzka, T. Boczar, P. Frącz, Acta Phys. Pol. A 120, 767 (2011).

[14] A. Cichoń, S. Borucki, D. Wotzka, M. Szmajda, Acta Phys. Pol. A 122, 804 (2012).

[15] S. Borucki, T. Boczar, A. Cichoń, Arch. Acoust. 32, Supplement, 291 (2007). 